\title{
Full-SiC derotator optics for METimage: detailed design and early model results
}

\author{
E. Renotte, C. Bastian, A. Bernat, M. Bougoin, B. \\ Carlomagno, et al.
}

E. Renotte, C. Bastian, A. Bernat, M. Bougoin, B. Carlomagno, C. Flebus, A. Feutry, E. Gillain, P. Gloesener, A. Jago, D. Logut, F. Mallet, Y. Martin, M. Pfaffe, A. Radioti, J.-M. Schumacher, F. Wolfs, "Full-SiC derotator optics for METimage: detailed design and early model results," Proc. SPIE 11852, International Conference on Space Optics - ICSO 2020, 1185256 (11 June 2021); doi: 10.1117/12.2599933

SPIE Event: International Conference on Space Optics - ICSO 2021, 2021, Online Only 


\section{International Conference on Space Optics-ICSO 2020}

Virtual Conference

30 March-2 April 2021

Edited by Bruno Cugny, Zoran Sodnik, and Nikos Karafolas
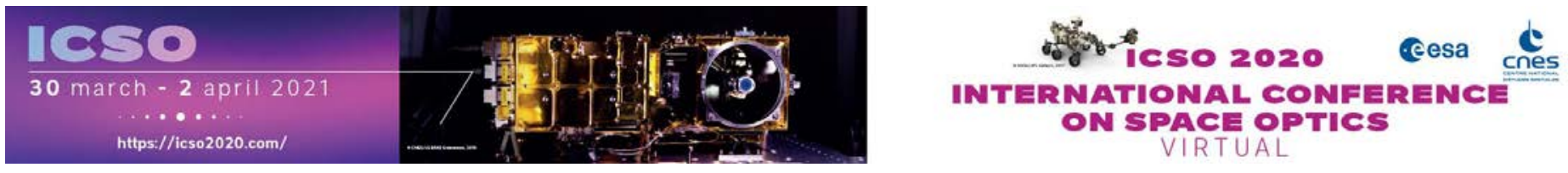

\section{Full-SiC derotator optics for METimage: detailed design and early model results}

\section{Cesa issopoceatings lecnes}




\title{
Full-SiC Derotator Optics for METimage: Detailed Design and Early Model Results.
}

\author{
E. Renotte ${ }^{\text {a }}$, C. Bastin ${ }^{\text {a }}$, A. Bernat ${ }^{\text {a }}$, M. Bougoin ${ }^{\text {d }}$, B. Carlomagno ${ }^{\text {a }}$, C. Flebus ${ }^{\text {a }}$, A. Feutry ${ }^{\text {a }}$, E. \\ Gillain $^{\text {a }}$, P. Gloesener ${ }^{\text {a }}$, A. Jago ${ }^{\text {b }}$, D. Logut ${ }^{\text {c }}$, F. Mallet ${ }^{\text {d }}$, Y. Martin ${ }^{\text {a }}$, M. Pfaffe ${ }^{\text {b }}$, A. Radioti ${ }^{\text {a }}$, \\ J-M. Schumacher ${ }^{\text {a }}$, F. Wolfs ${ }^{\text {a }}$. \\ ${ }^{a}$ AMOS s.a., Liège, Belgium ; ${ }^{b}$ Airbus Defence and Space GmbH, Immenstaad, Germany ; ${ }^{c}$ Airbus \\ Defence and Space SAS, Toulouse, France ; ${ }^{\mathrm{d}}$ Mersen Boostec SAS, Bazet, France
}

\begin{abstract}
METimage is an advanced multispectral radiometer for weather and climate forecasting developed by Airbus Defence \& Space under the auspices of the German Space Administration (DLR) for the EUMETSAT Polar System -Second Generation (EPS-SG). The instrument is equipped with a continuously rotating scan mirror with a $1.7 \mathrm{~s}$ period followed by a static telescope. The scan mirror permits an extended Earth view of $108^{\circ}$ per revolution and regular views to on-board calibration sources. A derotator assembly, which is half-speed synchronised with the scanner, is inserted in the optical beam after the telescope to compensate the image rotation in the focal plane. The derotator optical arrangement is a fivemirror concept that minimises the polarisation sensitivity. The derotator design is constrained by optical performance, mass and compactness, which led to the selection of a full silicon carbide ( $\mathrm{SiC}$ ) concept. This paper is presented as a continuation of the ICSO 2018 paper to describe the detailed design and early physical model results of the derotator optics.
\end{abstract}

Keywords: Earth observation, MetOp-SG, METimage, optical derotator, silicon carbide

\section{INTRODUCTION}

The EUMETSAT Polar System - Second Generation (EPS-SG) shall provide global observations from which information on variables of the atmosphere and the ocean and land surfaces can be derived. The observation data shall cover a broad spectral range (from UV to MW), are related to different spatial coverage (global and regional) and are characterised by a variety of different time scales, in order to continue and enhance the services offered by the EPS system. The EPS-SG mission encompasses various observation missions and consists of space and ground-based elements.

The Meteorological Operational Satellite - Second Generation (MetOp-SG) is the space segment of the EPS-SG mission. It is composed of two separate satellites, each carrying a different payload instruments complement (Fig. 1).

These satellites are operating in a low-earth, near-polar, sun-synchronous orbit with a midmorning mean local solar time descending node. They are 3-axis stabilised and Nadir-pointing with a yaw steering mode.

METimage is embarked on MetOp-SG satellite A. METimage [2,3] is implemented as passive imaging spectro-radiometer, capable of measuring thermal radiance emitted by the Earth and solar backscattered radiation in 20 spectral bands from $443 \mathrm{~nm}$ to $13.345 \mu \mathrm{m}$. The instrument achieves global coverage with $500 \mathrm{~m}$ square pixels by continuous scanning orthogonal to the flight direction. It employs in-field separation of the spectral channels. Due to the scan motion, the image moves sequentially over the detector channels. By proper timing of the sampling, a certain pixel in the image is measured sequentially by different spectral channels. The definition of the spectral range for the spectral bands is performed by filters in front of the detectors. The instrument is implemented as in-beam scanner with static telescope and synchronous field de-rotation. Calibration is performed during each scan with different calibration sources without interrupting the scientific observation. The observation principle is depicted in Fig 2. 


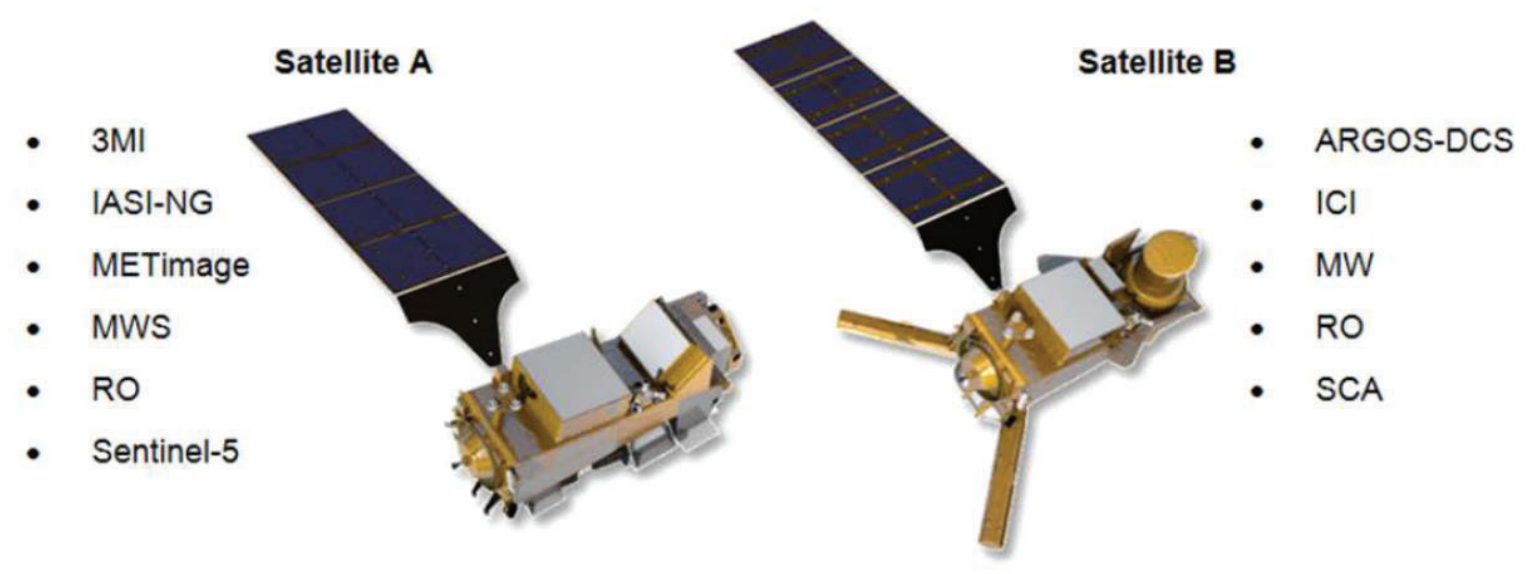

Figure 1. MetOp-SG, the two-satellite space segment of EUMETSAT's EPS-SG mission.

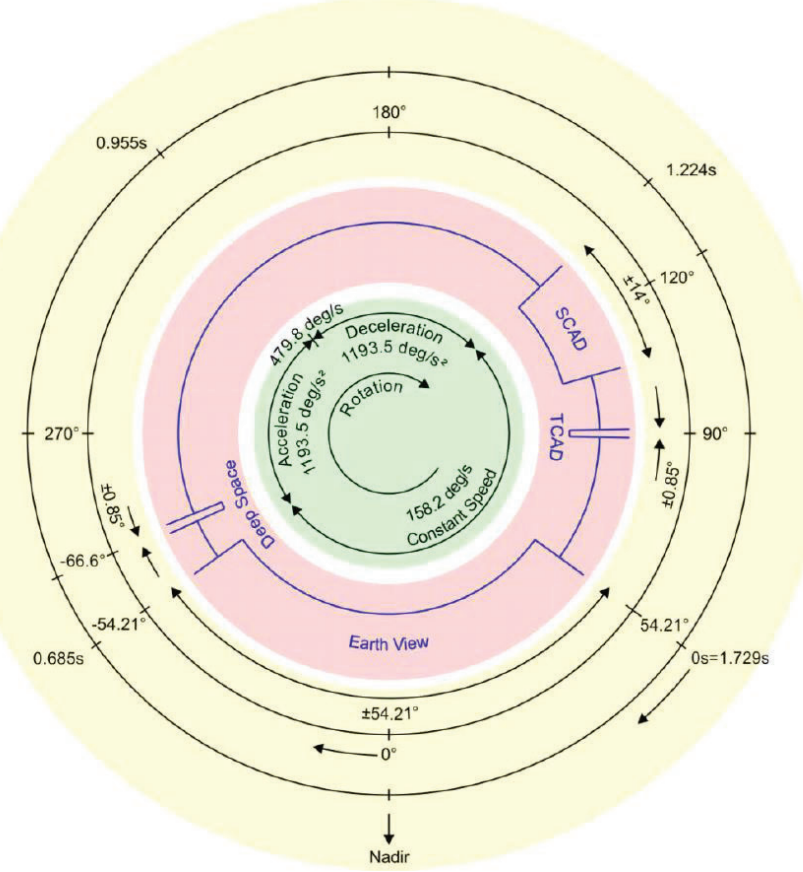

Figure 2. METimage observation principle.

At the entrance of the optical instrument a continuously rotating scan mirror is redirecting the light to the telescope, where the light either is coming from the Earth view or from the calibration sources. A de-rotator assembly, which is synchronised with the scanner and rotates at exactly half of the scan speed, follows the telescope and ensures a regular imaging geometry by correcting the image rotation in the focal plane. Two beam splitters split the observational wavelength range into three bands, each supported by a separate detector. The VNIR FPA is located in the telescope's focal plane. The 6 spectral bands are realised by filters. While the instrument and the visible focal plane operate at ambient temperature, the infrared optics and focal planes operate within a cryostat at $60 \mathrm{~K}$. Field masks within the optical paths of the infrared bands ensure proper spatial co-registration between the bands. The relay optics, needed to reduce the spot size at the infrared detectors, are implemented by lens optics. The infrared focal planes (SMWIR and LVWIR bands) are actively cooled by a pulse tube cooler. 


\section{DESIGN DESCRIPTION}

\subsection{Derotator Overview}

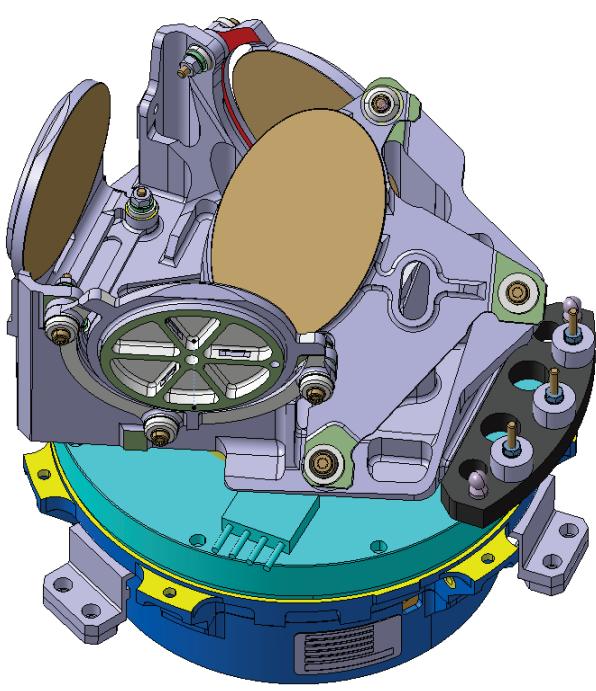

Figure 3. METimage Derotator Assembly (including mechanism, courtesy of Airbus Defence \& Space).

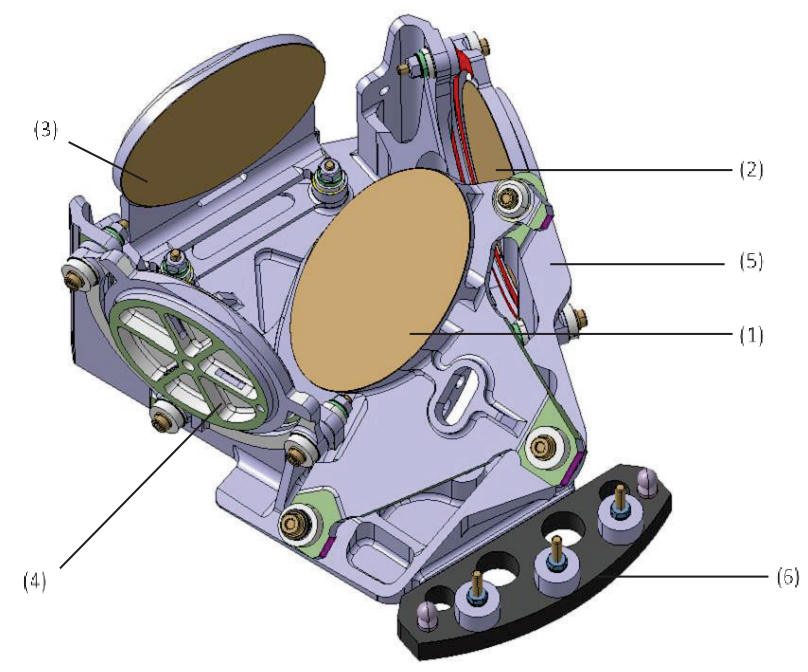

Figure 4. Derotator Optical Assembly.

The optical system consists of five flat mirrors (referred to as M1, M2, M3, M4 and M5), which form an optical derotator as originally presented in the US Patent 4,929,040 [1]. The derotaror optics is mounted on a rotating mechanism (Fig. 3) provided by Airbus Defence \& Space. During operation, the image of the object through the derotator rotates twice with the rotation of the derotator optics. The Derotator mechanism being half-speed synchronized with the scanner compensates the image rotation in the focal plane.

The orientation of the five mirrors with respect to each other is optimized to minimize polarization sensitivity. The five mirrors are made of BOOSTEC ${ }^{\circledR} \mathrm{SiC}$ material ( $\mathrm{SiC}$ ) and mounted on a baseplate from the same material. The baseplate is bonded to an Invar ring that mounts to the rotor of the mechanism.

The Derotator Optics is shown in Fig. 4. Its mass is $2.260 \mathrm{~kg}$ (calculated) with a maximum dimension of $254 \mathrm{~mm}$. It consists of:

- One structural baseplate (5) made of SiC material bonded to the interface ring of the Derotator mechanism (not shown in Fig. 4). A counterweight (6) is added to the baseplate for mass balancing,

- $\quad$ One M1-M5 duplex flat mirror (1) made of SiC material,

- $\quad$ M2 (2), M3 (3) and M4 (4) flat mirrors made of SiC material. 


\subsection{Baseplate}
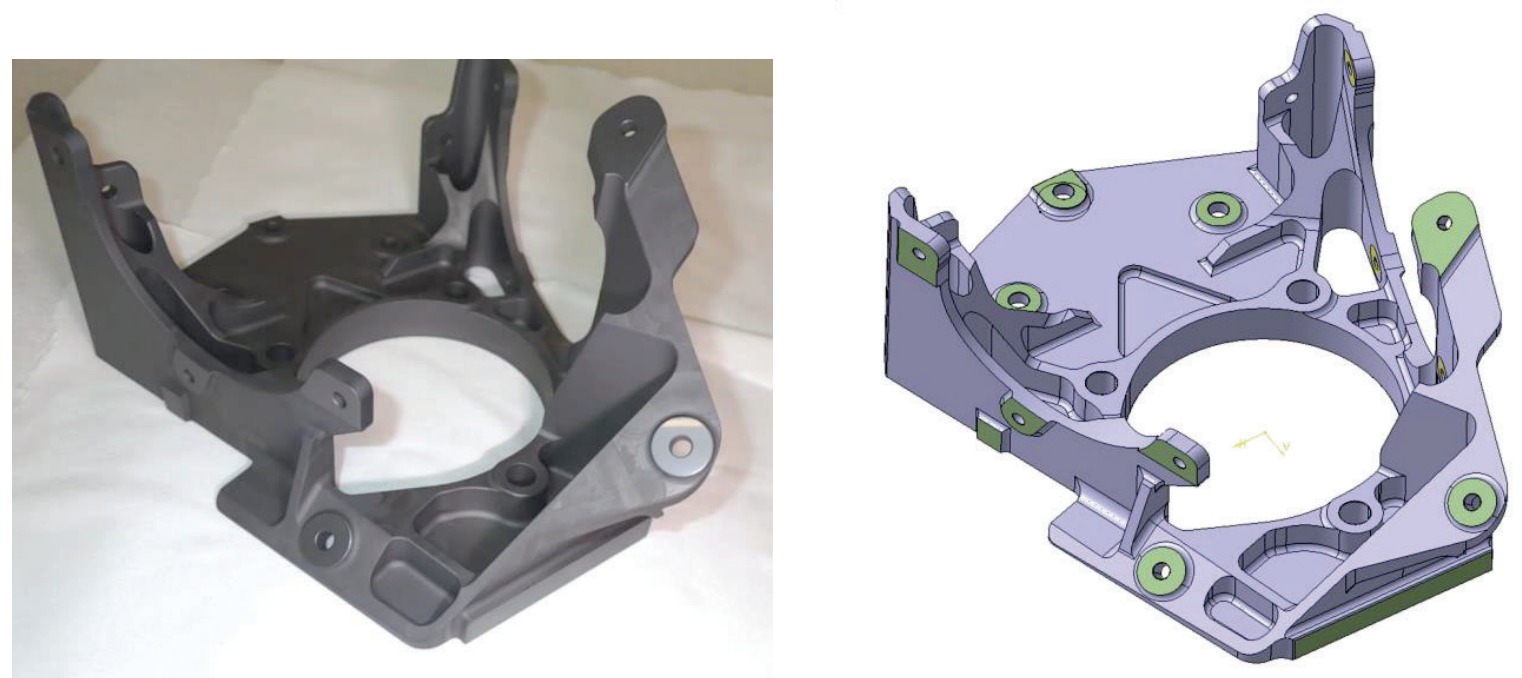

Figure 5. Derotator Baseplate.

The Baseplate is a monolithic piece of $\mathrm{SiC}(0.8 \mathrm{~kg})$ that provides four patterns of mounting holes for the individual mirrors, a circular entrance port on the mechanism side, and a pattern of three holes for interfacing the mechanism rotor on its bottom face (Fig. 5). An invar counterweight is added to the baseplate for mass balancing of the derotator around the rotation axis. The counterweight additional supports removable alignment references like e.g. CMM reference balls.

\subsection{Mirrors and Coating}

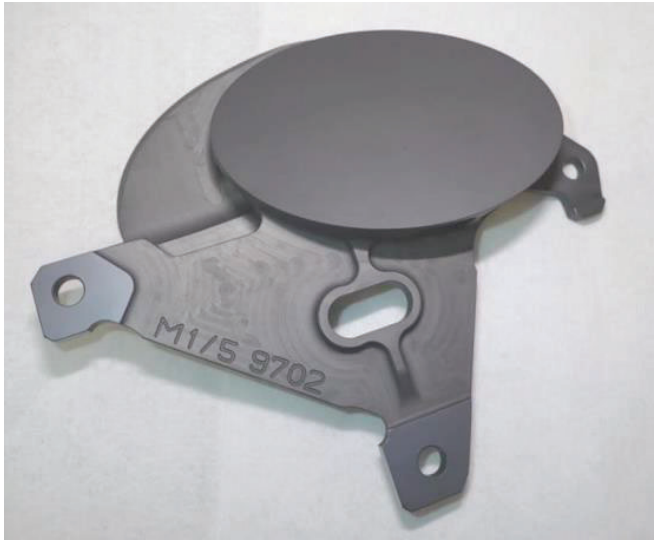

(a)

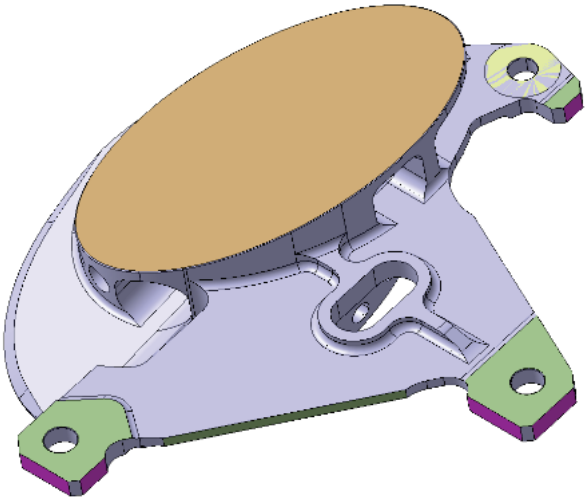

(b) 


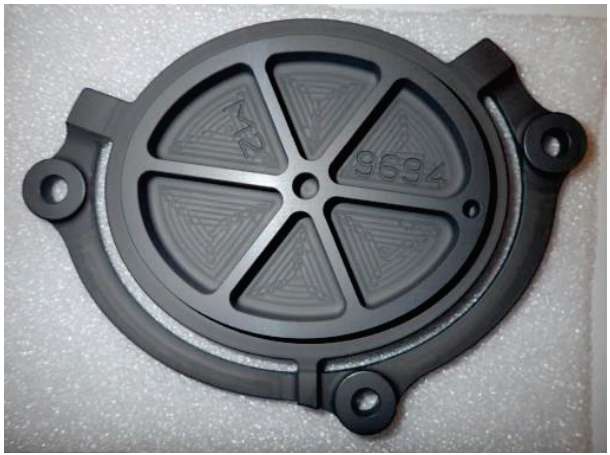

(c)

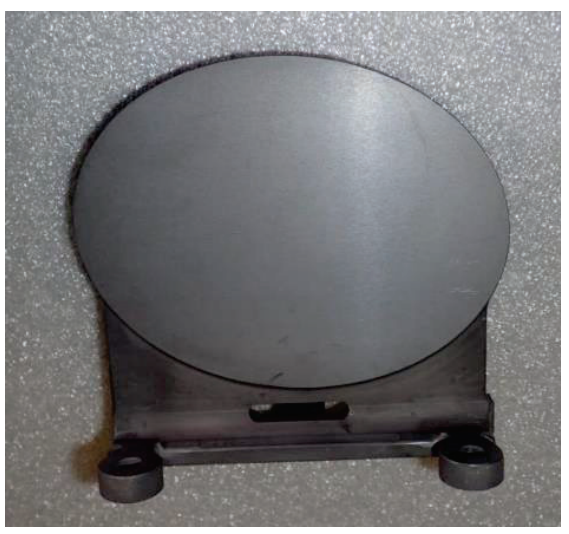

(e)

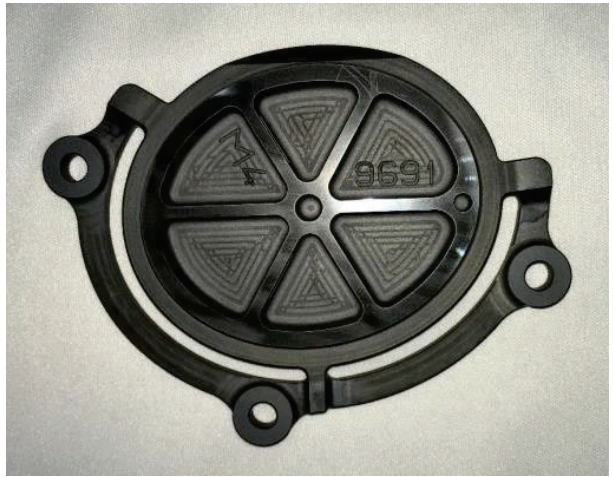

(g)

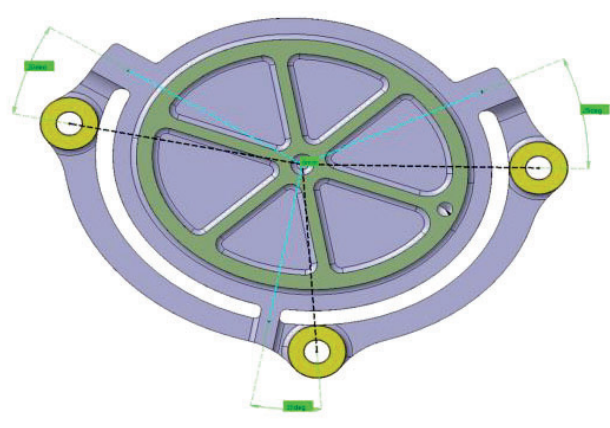

(d)

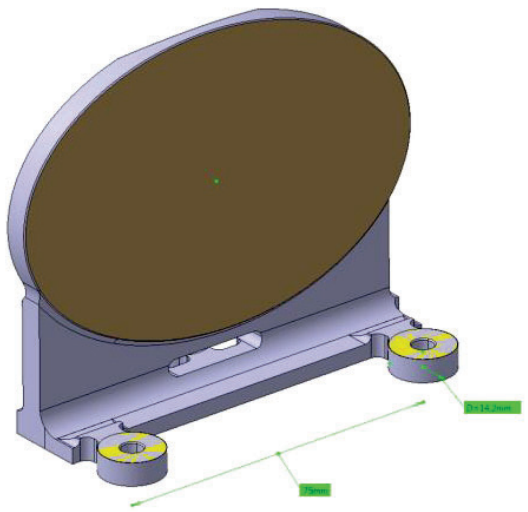

(f)

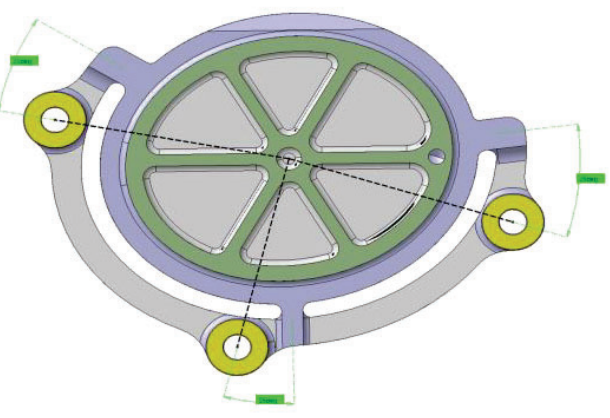

(h)

Figure 6. Mirror details: (a, b) M1/M5 duplex mirror, (c, d) M2, (e, f) M3, (g, h) M4.

Details of the mirror constructions (before optical polishing and coating) are shown in Fig. 6. M2 and M4 mount to the baseplate walls using a horseshoe shaped ring. Because of room restrictions, M3 rather uses a bracket to mount onto the baseplate bottom. M1 and M5 are arranged back to back, with a small prism angle, in a monolithic SiC piece. M1/M5 duplex mirror is mounted on a tilted plane. 
The optical surfaces of the mirrors are treated with Chemical Vapour Deposition (CVD) silicon carbide cladding. Per process, the CVD SiC is free of voids and can be optically polished. After polishing, a specific optical coating is applied on the mirror surfaces to improve the reflectivity and limit the polarization sensitivities. A protected silver coating with superior performance over the full wavelength range of METimage has been designed and space qualified for this programme.

Each mirror is fastened to the baseplate with three bolts. Except for M1/M5, shims are inserted between the mirror and the baseplate. The shims are adjusted for correcting planarity defects and aligning the mirrors (see below).

\section{OPTICAL PERFORMANCE}

The derotator optical performance requirements can be split in 3 categories

- Line-Of-Sight (LoS) performance (pointing, co-registration/distortion and pupil position), which are mainly linked to rigid body motion of individual mirrors

- Optical image quality (Wave Front Error), which is mainly linked to surface errors of individual mirrors

- Optical transmission and polarization, which are linked to the coating performance

LoS and image quality performance requirements are broken down by error source, and by mirror, as shown in next figure.

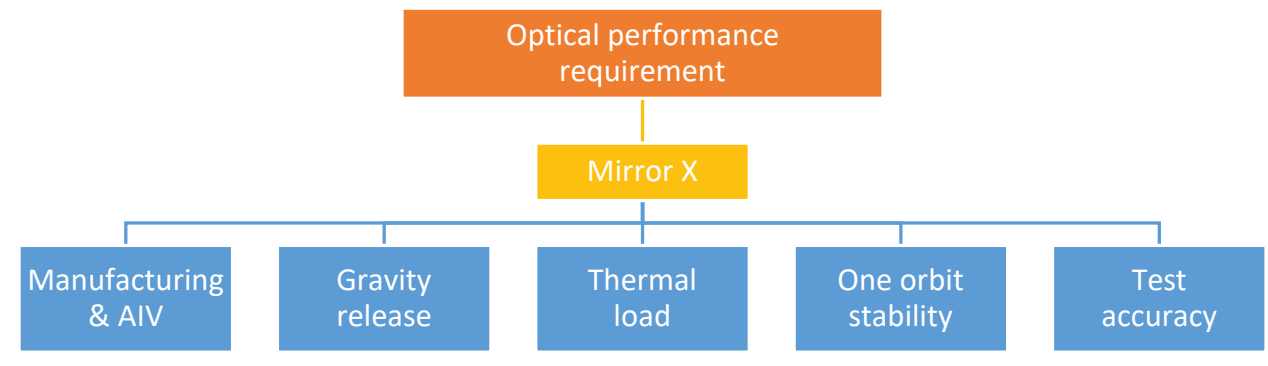

Figure 7. Optical performances error tree.

One particularity of this derotator is that the optical beam is not collimated but convergent, as shown on Figure 8 . This non-collimated beam induces different footprint on the different mirrors, as shown in Figure 9. The sensitivity of each optical performance to the position and surface figure error of each mirror is thus not direct and shall be evaluated from the optical model. Final performance is obtained by combining these optical sensitivities with the mirror displacement/surface error computed by finite element analysis or specified to manufacturing (polishing) and alignment. 


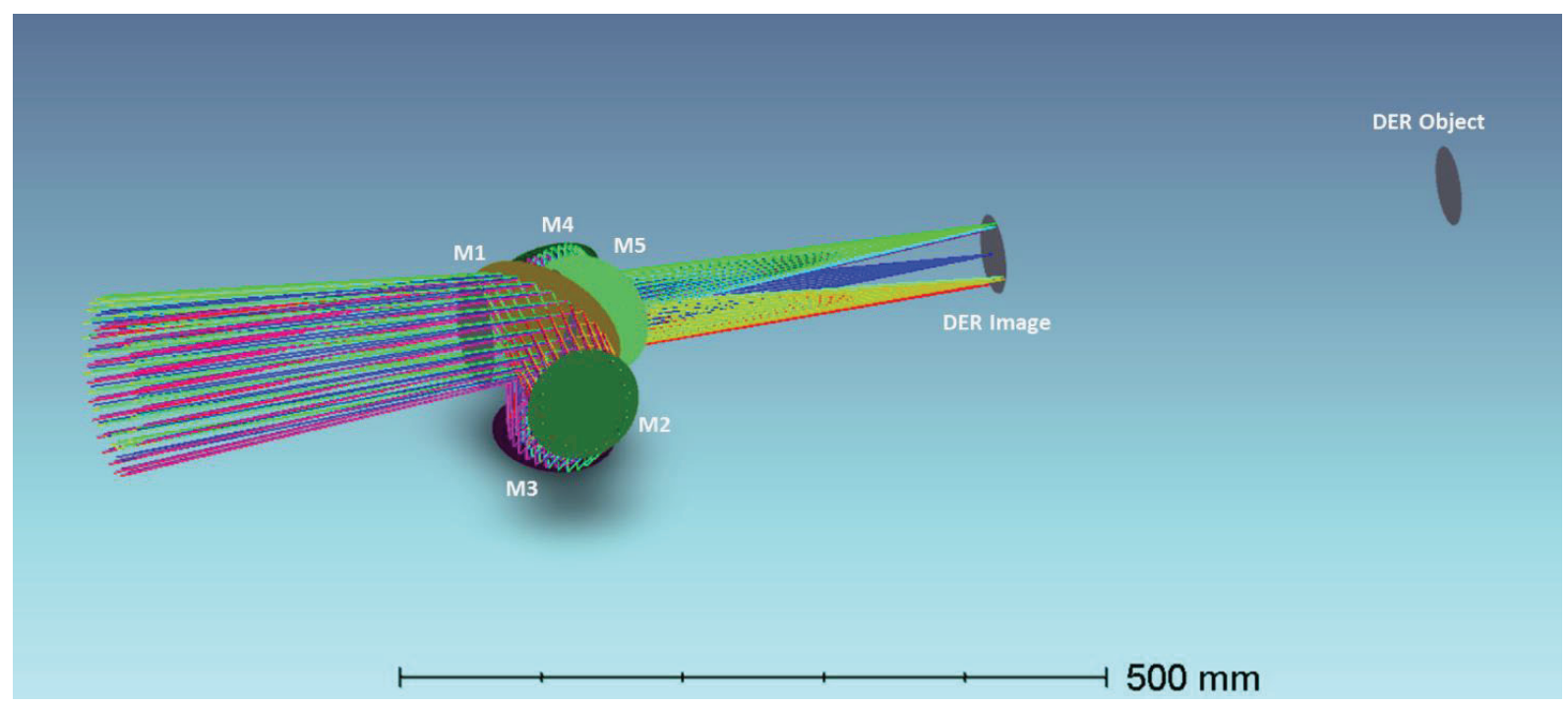

Figure 8. Optical design.

One of the tightest requirement is the Los/pointing performance, which allows only very small tilt/decenter on each mirror (a few arcsec). Hopefully, the main contributors (manufacturing \& AIV) can be compensated by proper alignment between the derotator optics and the mechanism. The combination of the remaining contributors is in-line with the required performance.

Exit pupil wobbling cannot fully be compensated in the same way that the pointing. However, the requirement is less severe $(170 \mu \mathrm{m}$ wobbling) and the desired performance is achieved with reasonable margins.

Image quality is specified in terms of wave front error (50 nm RMS) and wave front error gradient (7 $\mu$ rad RMS). This is quite tight for a set of 5 mirrors. The major contributors to these errors are obviously the polishing errors. The mechanical design of the mirrors, with integrated flexure, allows to keep the impact of integration errors to an acceptable level, and to reach the requested performance.
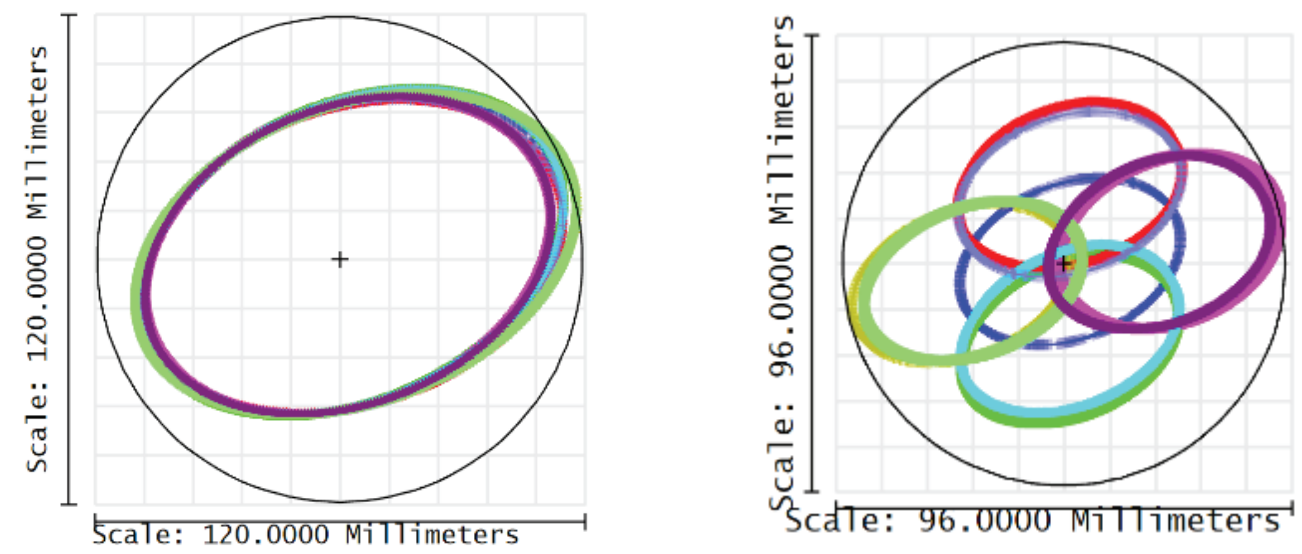

Figure 9. Footprint of different fields on M1 (left) and on M5 (right). 
Transmission and polarization requirements are mainly dependent on coating design and manufacturing, and reflectivity measurement accuracy. The proposed optical coating has been optimised to approach at best the mission requirements. Its end-of-life performance was assessed through a severe test programme.

\section{MECHANICAL PERFORMANCE}
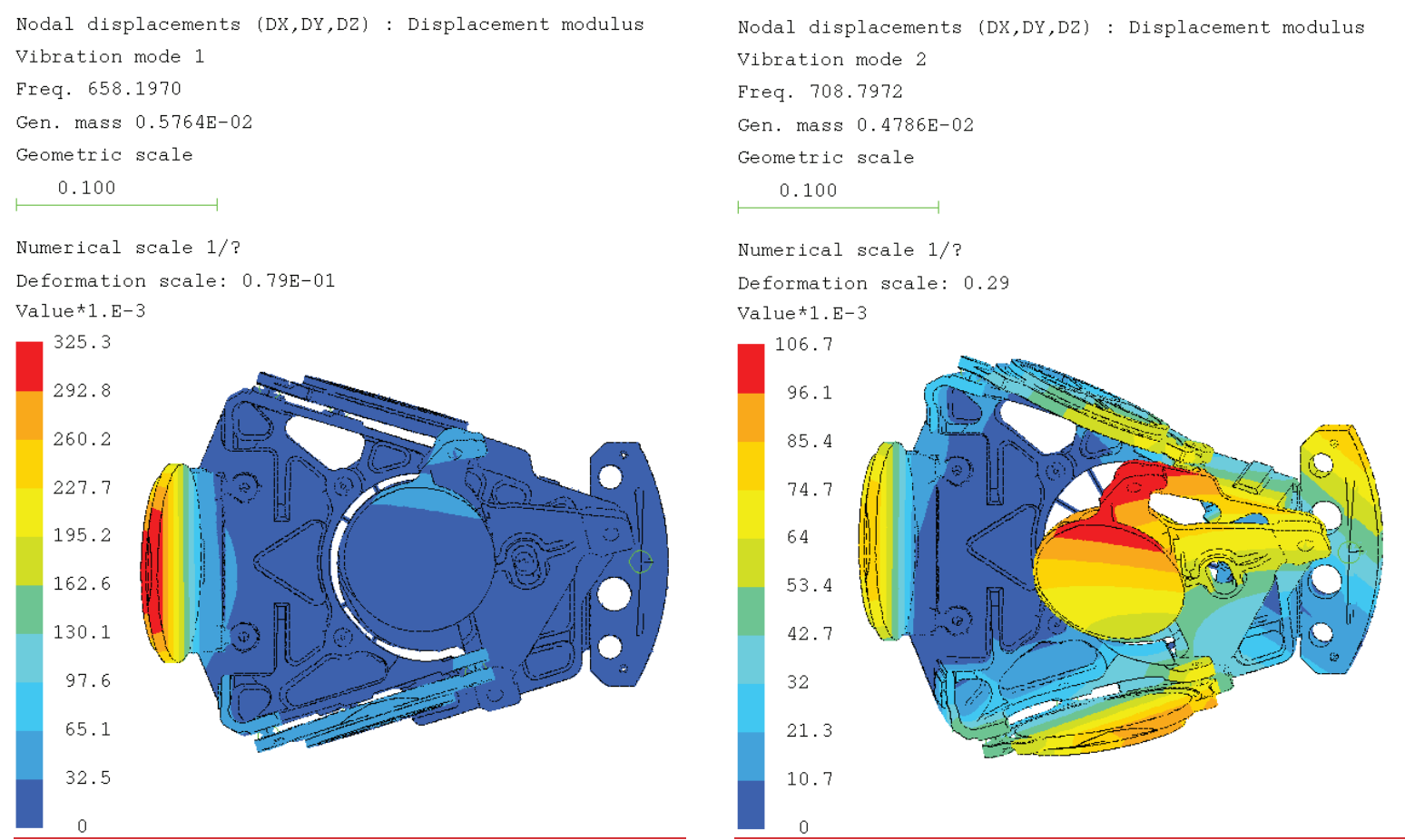

Figure 10. First two vibration modes at $658 \mathrm{~Hz}$ and $709 \mathrm{~Hz}$ evaluated by finite element modelling.

The proposed design has been modelled and verified for various load cases, operational and environmental constraints, including:

- $\quad$ Lifetime (up to 22 years on ground, plus 7.5 years in orbit)

- Mechanical envelope

- Mass properties (mass, moments of inertia, balance around rotation axis)

- Radiation hardness

- $\quad$ Stiffness (eigenfrequency)

- Gravity

- Mechanical loads (quasi-static loads, sine vibration, random vibration, shock)

- Integrity and stability of bolted joints

- Buckling

- Imposed displacements (resulting from imperfect contact surfaces)

- Thermo-elastic constraints (resulting from temperature changes and gradients)

- Venting of closed cavities

- Fatigue (damage tolerance)

The detailed analyses have demonstrated that the design is compatible with all physical requirements (mass, dimensions, stiffness...) imposed by the instrument accommodation. Fig. 10 shows the first two natural vibration modes $(658 \mathrm{~Hz}$, $709 \mathrm{~Hz}$ ) of the Derotator Optics. 
When exposed to mechanical loads, the shock and random vibration appear to be the most severe solicitations, which will require adequate notching of the input levels.

\section{DEVELOPMENT \& VERIFICATION APPROACH}

\subsection{Model Philosophy}

Table 1. Derotator model philosophy summary.

\begin{tabular}{|l|l|l|}
\hline \multicolumn{1}{|c|}{ Model } & \multicolumn{1}{|c|}{ Representativeness } & \multicolumn{1}{c|}{ Use } \\
\hline Structural \& Thermal Model (STM) & $\begin{array}{l}\text { Mechanical/thermal representatives, } \\
\text { not optical (no CVD, no coating) }\end{array}$ & $\begin{array}{l}\text { Assembly training, } \\
\text { I/F fit check, design qualification }\end{array}$ \\
\hline $\begin{array}{l}\text { Engineering Qualification Model } \\
\text { (EQM) }\end{array}$ & $\begin{array}{l}\text { Refurbished STM (semi-polished } \\
\text { mirrors, without CVD) }\end{array}$ & $\begin{array}{l}\text { To support instrument level } \\
\text { verifications }\end{array}$ \\
\hline Flight Model 1 (FM1) & full flight standard & $\begin{array}{l}\text { Flight use, to populate instrument } \\
\text { FM1 }\end{array}$ \\
\hline Flight Model 2 (FM2) & Identical to FM1 & $\begin{array}{l}\text { Flight use, to populate instrument } \\
\text { FM2 }\end{array}$ \\
\hline Flight Model 3 (FM3) & Identical to FM1 & $\begin{array}{l}\text { Flight use, to populate instrument } \\
\text { FM3 }\end{array}$ \\
\hline Flight Spare (FS) & Identical to FM1 & Replacement of faulty unit as needed \\
\hline
\end{tabular}

The STM is based on the flight design but the optical surfaces are not optically finished. i.e. no CVD SiC, no mirror figuring, no mirror polishing, no optical coating, no alignment. The STM is intended for AIT training, interface fit-checks and design qualification. The STM shall be submitted to full mechanical \& thermal qualifications of the assembly including the glued interface ring.

The EQM shall reuse the STM hardware with refurbished mirrors polished to roughly $5 \mathrm{~nm}_{\mathrm{RMS}}, 2$ fringes and coated (without CVD). The Derotator Optics EQM shall be aligned to flight standard and shall be submitted to thermal and mechanical acceptance (1-axis).

The Derotator Optics flight models (FM1, FM2, FM3) shall use qualified materials, parts and processes according to all configuration control and product assurance provisions. The Derotator Optics FM2 \& FM3 shall be identical copies of the FM1. The FM1, FM2 and FM3 shall be submitted to the full sequence of acceptance testing prior to delivery.

The FS is intended to be a one-to-one replacement unit in case of accidental failure of a flight model.

\subsection{Assembly, Integration and Verification Overview}

A discussion of the AIV activities and GSE was presented in [4]. The AIV sequence is recalled in the following chart for reference. 


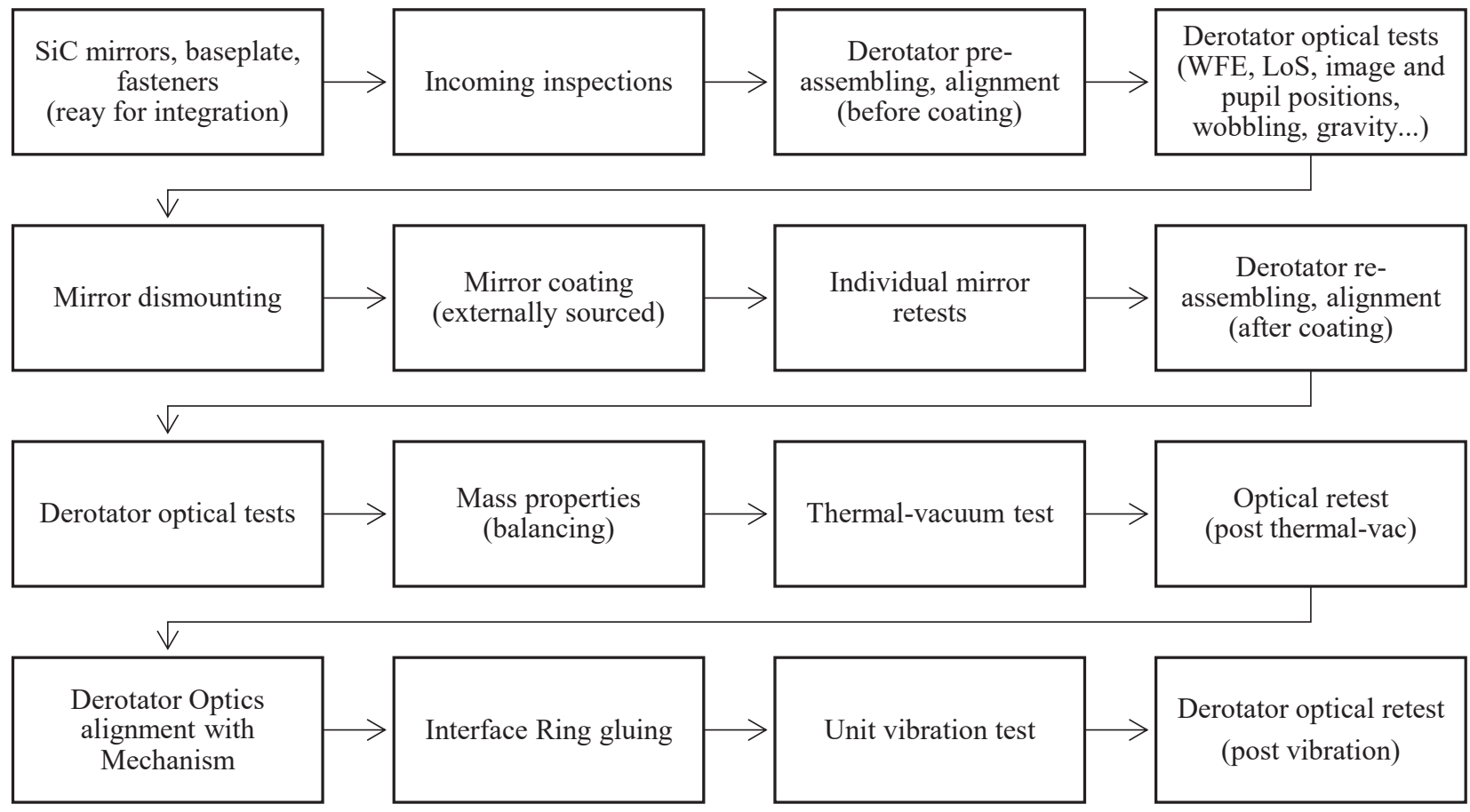

Figure 11: AIV Flow Chart (FMs).

\subsection{STM verification campaign}

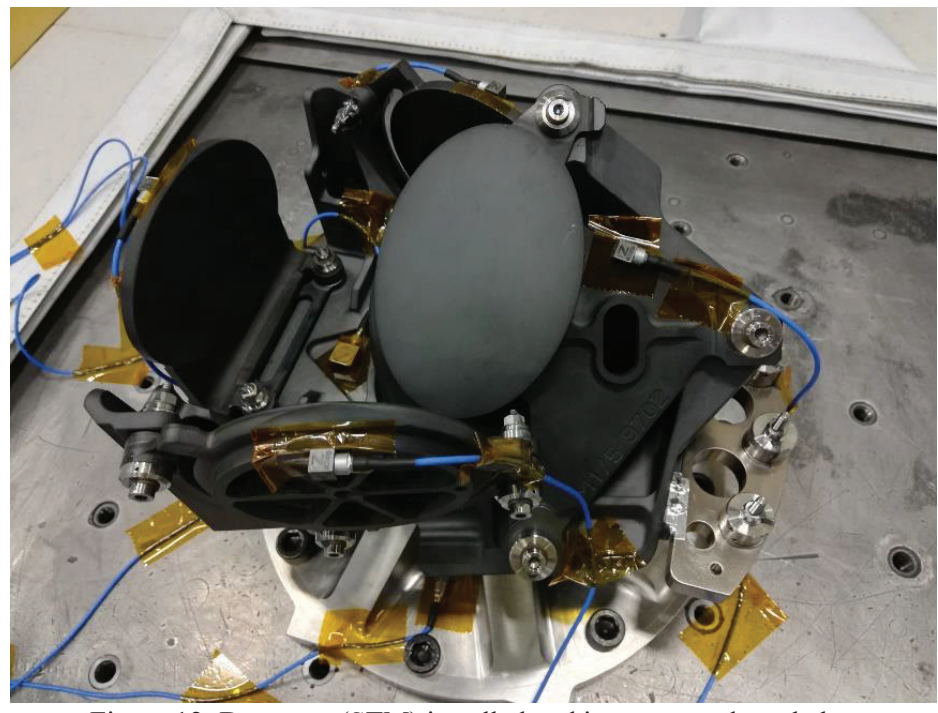

Figure 12: Derotator (STM) installed and instrumented on shaker table.

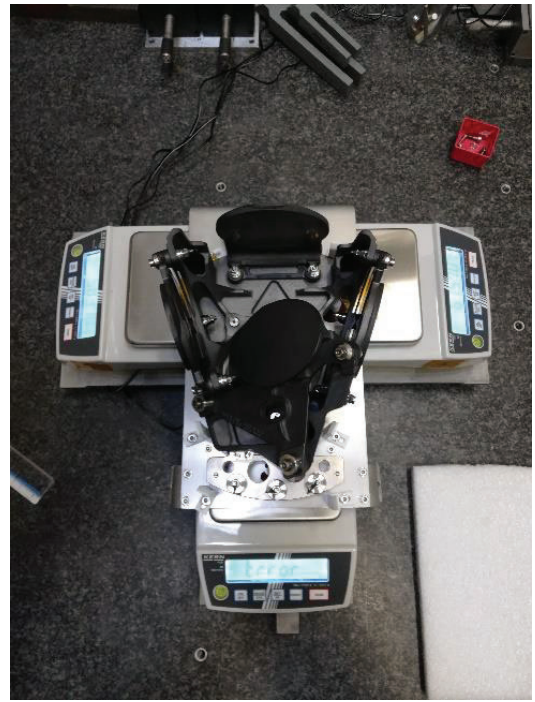

Figure 13: Derotator (STM) mass properties measurement. 
A full-scale, mechanically representative STM model of the derotator was built. It served two main objectives: $1^{\circ}$ ) a training / validation of the integration procedures and tools, and $2^{\circ}$ ) a verification of the mechanical design ability to survive qualification loads, including shocks, which cannot be conducted on actual flight hardware.

With regard to the compactness of the derotator design, the verification of the integration procedure was a key objective. The sequence was first experienced with a 3D-printed plastic model of the derotator, which provided useful learnings.

After successful integration, the STM was then submitted to the following test sequence:

- Initial metrology health check (with 3D CMM)

- Shock test

- Metrology re-check (with 3D CMM)

- Vibration test (frequency search, sine, random)

- Metrology re-check (with 3D CMM)

- Thermal-vacuum cycling (8 cycles between non-operational temperature limits + qualification margins)

- Metrology re-check (with 3D CMM)

- Dimensional verifications

- Mass properties (mass, CoG, balancing)

- $\quad$ Electrical properties

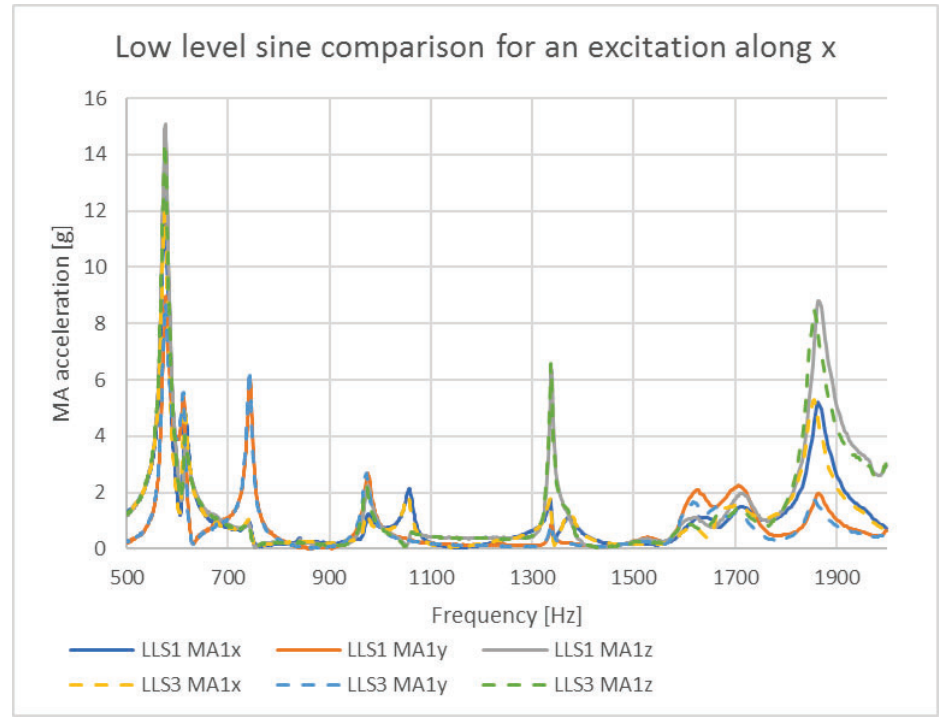

Figure 14: Derotator (STM) frequency search.

First, as shown on Fig.14, the mechanical tests showed a satisfactory matching with finite-element modelling predictions. Besides minor acceptable deviations, the STM verification programme has been declared successful and validated the mechanical and thermal design, as well as the integration procedure and set-ups.

\section{FUTURE WORK}

This paper is presented after the completion of the CDR and STM programme. The STM was dismounted to be refurbished as an EQM. This consists of upgrading the mirrors with a reduced optical quality. The first two flight models are in production (mirror polishing) and will assembled and tested within the coming year. 


\section{CONCLUSIONS}

This paper presented the detailed design and early test model results of a full-SiC five-mirror derotator for the METimage instrument. The $\mathrm{SiC}$ design has been preferred over other options because of its compactness, mass and robustness. The proposed design fulfills the accommodation constraints in terms of mounting interface, mass, envelope and stiffness. It satisfies the optical performance requirements. It also satisfies the thermal and mechanical requirements as demonstrated by the STM test campaign.

\section{ACKNOWLEDGEMENTS}

The METimage Intrument described herein will be developed by an industrial team led by Airbus Defence and Space $\mathrm{GmbH}$ on behalf of the German Space Administration DLR with funds from the German Federal Ministry of Transport and Digital Infrastructure and co-funded by EUMETSAT under DLR Contract No. 50EW1521. The Derotator Optics are developed by AMOS s.a. for Airbus Defence \& Space GmbH under R\&D contract No. F.45706/G01000-6593.

\section{REFERENCES}

[1] T. Pagano, R. Turtle; "Five mirror, two perpendicular plane image derotator", US Patent 4,929,040 (29 May 1990).

[2] O.Wallner, T. Reinert and C. Straif, "METimage - A Spectro-Radiometer for the VII Mission onboard MetOpSGS", Proc. SPIE 10562, International Conference on Space Optics - ICSO 2016.

[3] X. Gnata, M. Weidinger, F. Schmülling, "Metimage: calibration and performance verification", Proc. SPIE 10562, International Conference on Space Optics - ICSO 2016.

[4] E. Renotte et al. "Full-SiC Derotator Optics for METimage: Preliminary Design and Verification Approach", Proc. SPIE 11180, International Conference on Space Optics — ICSO 2018. 